\title{
Weitere Beiträge über das Vorkommen von Betainen im Pflanzenreich.
}

Von

K. Yoshimura und G. Trier.

(Aus dem agrikulturchemischen Laboratorium der Eidgenössischen Technischen Hochschule in Zürich.)

(Der Redaktion zugegangen am 8. März 1912)

Die vorliegende Arbeit bildet eine Fortsetzung der in den letzten Jahren im hiesigen Institut ausgeführten Untersuchungen über die in den Pflanzen vorkommenden Betaine. ${ }^{1}$ ) Wie in der letzten Publikation bemerkt worden ist, ist das Auftreten der Betaine ein sporadisches. Man wird im allgemeinen innerhalb einer Pfanzenfamilie auf das Vorkommen gleicher oder ähnlicher Betaine nur rechnen können, wenn man Pflanzen untersucht, die sich im natürlichen System sehr nahe stehen. ${ }^{2}$ ) So wurde schon mitgeteilt, daß Betaine (Stachydrin, Betonicin, Trigonellin) in der Familie der Labiaten zwar in mehreren Arten der Gattung Stachys aufgefunden wurden, nicht aber bei Salvia pratensis, die einer anderen Untergruppe der Stachyoideae angehört. ${ }^{3}$ ) Wir können nun ergänzend mitteilen, daß wir auch bei Glechoma

1) E. Schulze und G. Trier, Diese Zeitschrift, Bd. 67, S. 46 und 59. - Bd. 76, S. 258. - E. Schulze und U. Pfenninger, Diese Zeitschrift, Bd. 71, S. 174 .

2) Staněk und Domin (Zeitschrift für Zuckerindustrie in Böhmen, Bd. 34, S. 297, 1910) fanden das Betain, $\mathrm{C}_{5} \mathrm{H}_{11} \mathrm{NO}_{2}$, in allen darauf hin untersuchten Chenopodiaceen, sowie in zwei Gattungen der nahe verwandten Amarantaceen. Dagegen fanden sie Betain nicht in anderen Familien der Reihe Centrospermae. Als Vertreter der dieser Reihe angehörenden Nyctaginaceen untersuchten sie Mirabilis jalapa. Wie weiter unten angegeben ist, konnten wir in dieser Pflanze Trigonellin nachweisen.

3) Siehe Englers Syllabus der Pflanzenfamilien. 
Beiträge über das Vorkommen von Betainen im Pflanzenreich. 291

hederacea L., die dem Tribus der Nepeteae innerhalb der Unterfamilie der Stachyoideae zugezählt wird, kein Betain aufzufinden vermochten. Dagegen fanden wir Stachydrin, und zwar in der bisher noch nicht beobachteten optisch aktiven, linksdrehenden Form in der den Stachysarten sehr nahe stehenden Galeopsis ochroleuca Lam. In zwei Pflanzen, die der Unterfamilie der Ajugoideae angehören, nämlich Ajuga reptans L. und Rosmarinus officinalis L., fanden sich dagegen wieder keine Betaine.

Das Stachydrin war bisher außer bei Labiaten nur in Blättern der bitteren und der süßen Orange (Citrus aurantium L., Familie der Rutaceae, Unterfamilie der Aurantioideae) nachgewiesen worden. Wir fanden es auch in Citronenschalen. In Blättern von Fagara xanthoxyloides Lam., ${ }^{1}$ ) die der Unterfamilie der Rutoideae zugezählt wird, fanden wir kein Anzeichen für das Vorhandensein eines Betains.

Dagegen vermochten wir Stachydrin in einer weiteren Familie, nämlich unter den Kompositen, nachzuweisen. Es zeigte sich nämlich, daß das aus dem sogenannten *dalmatinischen Insektenpulver", den gepulverten Blüten von Chrysanthemum cinerariifolium Bocc. von Marino-Zucco²) erhaltene Alkaloid Chrysanthemin keine einheitliche Verbindung ist, sondern aus einem Gemisch besteht, aus welchem wir Cholin und Stachydrin zu isolieren vermochten.

Schließlich fanden wir bei der der Familie der Nyctaginaceen angehörenden Mirabilis jalapa L. das so weit verbreitete Trigonellin.

In allen mit genügend Material daraufhin untersuchten

1) Wir verdanken eine Probe von $670 \mathrm{~g}$ der Blätter der großen Liebenswürdigkeit von Herrn Prof. Dr. H. Thoms in Berlin. Über die von Prof. Thoms und seinen Mitarbeitern in Fagara xanthox. entdeckten interessanten Pflanzenstoffe Xanthotoxin und Fagaramid siehe Berichte d. Deutsch. chem. Ges., Bd. 44, S. 3325 und S. 3729. - ApothekerZeitung, Bd. 26, S. 1043. - Biochem. Zeitschrift, Bd. 38, S. 492.

2) Marino-Zucco, Atti della Reale Accad. dei Lincei (4), 5. I., S. 527. - 6. I., S. 571. - 7. I., S. 121. - (5), 4. I., S. 247.

Gazzetta chimica italiana, Bd. 19, S. 209. - Bd. 21, S. 516. Bd. 25, 1., S. 257. 
Objekten (Chrysanthemum, Ajuga, Rosmarinus, Glechoma, Galeopsis) konnte ferner Cholin nachgewiesen werden.

I. Nachweis von Cholin und Stachydrin in den Blüten von Chrysanthemum cinerariifolium Bocc. (Insektenpulver).

Im Jahre 1890 isolierte Marino-Zucco aus den Blüten der bezeichneten Chrysanthemumart, die zur Bereitung von Insektenpulver verwendet werden, eine basische Verbindung, der er auf Grund umfassender Studien die folgende Konstitution zuschrieb :

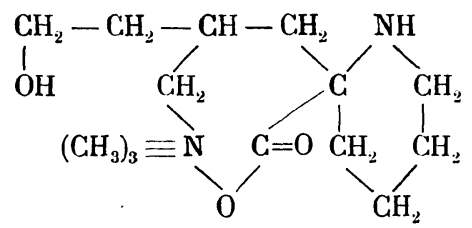

Es war vorauszusehen, daß diese Auffassung nicht ganz richtig sein konnte und zwar schon aus dem Grunde, weil nach der Darstellungsweise des Entdeckers das von ihm untersuchte Präparat nicht einheitlich sein konnte und jedenfalls auch Cholin einschloß. Wir vermuteten ferner das Vorhandensein von Betainen.

Wir gingen von $4 \mathrm{~kg}$ der im Handel erhältlichen *Flores Chrisanthemi cinerariifolii pulvis subt. aus besten wildgewachsenen, geschlossenen Gebirgsblüten» aus. Davon verarbeiteten wir $2 \mathrm{~kg}$ nach einem Verfahren, das im wesentlichen mit dem von Marino-Zucco beschriebenen übereinstimmt. Die andere Hälfte wurde nach der im hiesigen Laboratorium gebräuchlichen, in früheren Mitteilungen beschriebenen Methode aufgearbeitet. In beiden Fällen erhielten wir allem Anscheine nach gleiche Produkte, aus welchem wir Cholin und inaktives Stachydrin isolieren konnten.

A. Aufarbeitung des Materials nach Marino-Zucco.

$2 \mathrm{~kg}$ Insektenpulver wurden mit warmem Wasser zweimal ausgezogen, die Extrakte mit Bleiessig gefällt, die Filtrate vom Bleiniederschlag durch Schwefelsäure vom Blei befreit und 
Beiträge über das Vorkommen von Betainen im Pflanzenreich. 293

dann mit Kaliumwismutjodidlösung (nach Kraut) versetzt. Der rote Niederschlag wurde nach dem Auswaschen mit schwefelsäurehaltigem Wasser mit Bleicarbonat zerlegt, das durch Schwefelwasserstoff vom Blei befreite Filtrat mit feuchtem Silberoxyd vom Jod völlig befreit und schließlich das Silber durch Salzsäure entfernt. Die erhaltene Lösung der Chloride wurde im Wasserbad eingedunstet, der dunkelgefärbte Sirup mit Tierkohle gereinigt, wieder eingeengt und im Vakuumexsikkator stehen gelassen, wobei allmählich hygroskopische salzsaure Salze auskrystallisierten.

Ein kleiner Teil der Chloride wurde in das Chloraurat übergeführt, welches im Kapillarrohre bei $239-240^{\circ}$ unter Zersetzung schmolz.

Die Analyse des Aurats ergab:

$0,1964 \mathrm{~g}$ Substanz gaben 0,0821 g Au $=41,80 \% \mathrm{Au}$.

Ein anderer Teil der salzsauren Salze wurde in absolutem Alkohol gelöst, mit alkoholischer Sublimatlösung ausgefällt und die erhaltenen Quecksilberdoppelsalze mit Schwefelwasserstoff zerlegt. Die so erhaltenen Chloride wurden nach Stanð̌ks Verfahren mit Perjodid behandelt.

Das in alkalischer Lösung gefällte Perjodid (*Cholinfraktion*) wurde in das Chlorhydrat und dieses in das Chloraurat übergeführt. Letzteres schmolz im Kapillarrohr bei $265^{\circ}$ unter Zersetzung.

0,0784 g Substanz gaben 0,0348 g Gold.

Für Cholinchloraurat $\mathrm{C}_{5} \mathrm{H}_{14} \mathrm{NOCl} \cdot \mathrm{AuCl}_{3}$

Berechnet:

$44,50 \% \mathrm{Au}$.
Gefunden :

$44,39 \% \mathrm{Au}$.

Das in saurer Lösung gefällte Perjodid («Betainfraktion») wurde ebenfalls in das Chlorhydrat übergeführt. Es zeigte deutliche Pyrrolreaktion. Das Chloraurat zeigte unter dem Mikroskop die charakteristische Form des Stachydringoldsalzes. Im Kapillarrohr erhitzt schmolz es bei 225-226 .

0,3334 g Substanz gaben 0,1352 g Au.

Für Stachydrinchloraurat $\mathrm{C}_{7} \mathrm{H}_{13} \mathrm{NO}_{2} \cdot \mathrm{HCl} \cdot \mathrm{AuCl}_{3}$

Berechnet:

$40,82 \% \mathrm{Au}$.
Gefunden:

40,52\% Au. 
B. Aufarbeitung des Materials nach dem von E. Schulze angewendeten Verfahren. ${ }^{1}$ )

$2 \mathrm{~kg}$ Insektenpulver wurden mit warmem Wasser extrahiert, die Auszüge mit Bleiessig gereinigt, dann mit Schwefelsäure entbleit und mit Phosphorwolframsäure ausgefällt. Der Niederschlag wurde in bekannter Weise mit Baryt zersetzt, die erhaltene Lösung der freien Basen mit Salpetersäure neutralisiert, dann mit Silbernitrat und Silbernitrat und Baryt gefällt. Das Filtrat von diesen Niederschlägen wurde mittels Salzsäure und Schwefelsäure von Silber und Baryt befreit, sodann wieder mit Phosphorwolframsäure gefällt. Die aus der Phosphorwolframsäurefällung regenerierte Basenlösung wurde mit überschüssiger Salzsäure angesäuert, stark eingeengt und im Vakuumexsikkator krystallisieren gelassen.

Die so erhaltene hygroskopische Krystallmasse wurde in zwei Hälften geteilt.

Die eine Hälfte der salzsauren Salze wurde in absolutem Alkohol aufgenommen, wobei fast alles in Lösung ging. Die alkoholische Lösung wurde mit alkoholischer Sublimatlösung gefällt. Die Quecksilberdoppelsalze wurden mit Schwefelwasserstoff zersetzt, die erhaltenen Chloride im Vakuumexsikkator auskrystallisieren gelassen und dann in das Chloraurat übergeführt.

0,6654 g Substanz gaben $0,2791 \mathrm{~g}$ Gold $=41,94 \% \mathrm{Au}$. Im Kapillarrohre erhitzt schmolz das Aurat bei $245^{\circ}$ unter Zersetzung.

Die andere Hälfte der salzsauren Salze wurde auf eine Tonplatte gestrichen und im Exsikkator längere Zeit stehen gelassen. So blieb der größte Teil der Salze auf der Tonplatte und war nach dem Herausnehmen an der Luft nicht mehr zerfließlich. (Ein 'Teil des salzsauren Salzes, der auf eine Tonplatte verstrichen wurde, die an der Luft verblieb, wurde vollkommen in die Tonplatte hineingezogen).

Der auf der Tonplatte zurückgebliebene Rŭckstand wurde mit ganz wenig absolutem Alkohol in der Kälte verrieben,

1) Diese Zeitschrift, Bd. 60, S. 155 . 
Beiträge über das Vorkommen von Betainen im Pflanzenreich. 295

wieder auf eine Tonplatte gestrichen und zuletzt aus Wasser umkrystallisiert. Es wurde so etwa $0,8 \mathrm{~g}$ eines Salzes erhalten, das sich als reines Stachydrinchlorhydrat erwies. Das Salz zeigte deutliche Pyrrolreaktion; im Kapillarrohr erhitzt schmolz es bei $233-235^{\circ}$.

Chloraurat: Das aus einem Teil des salzsauren Salzes dargestellte Chloraurat zeigte unter dem Mikroskop die charakteristische Form des Stachydringoldsalzes. Es schmolz bei $225^{\circ}$ unter Zersetzung.

$$
0,4220 \text { g Substanz gaben } 0,1717 \mathrm{~g} \text { Gold. }
$$

Für Stachydrinchloraurat $\mathrm{C}_{7} \mathrm{H}_{13} \mathrm{NO}_{2} \cdot \mathrm{HCl} \cdot \mathrm{AuCl}_{3}$.

Berechnet:

$40,82 \% \mathrm{Au}$.
Gefunden:

$40,69 \% \mathrm{Au}$.

Chloraurat des Äthylesters: Ein Teil des Stachydrinchlorhydrats wurde mit Alkohol und Salzsäure verestert und in das Chloraurat des Äthylesters übergeführt, welches auch in heißem Wasser ziemlich schwer löslich ist. Es schmolz bei $60^{\circ 1}$ ) und zersetzte sich bei $241-244^{\circ}$ unter Schäumen.

$0,2058 \mathrm{~g}$ Substanz gaben $0,0796 \mathrm{~g}$ Gold.

Für Stachydrinäthylesterchloraurat $\mathrm{C}_{9} \mathrm{H}_{17} \mathrm{NO}_{2} \cdot \mathrm{HCl} \cdot \mathrm{AuCl}_{3}$.

Berechnet:

Gefunden :

$38,58 \%$ Au.

$38,58 \% \mathrm{Au}$.

Pikrat des Stachydrins: ${ }^{2}$ ) Das durch Versetzen der wässerigen Lösung des salzsauren Stachydrins mit Natriumpikrat erhaltene Pikrat bildete gelbe Nadeln, welche im Aussehen und im Schmelzpunkt $\left(195^{\circ}\right)$ mit Stachydrinpikrat übereinstimmten.

Nachweis des Gholins. Die zur Abtrennung des Stachydrinchlorids benutzte Tonplatte wurde mit heißem Wasser ausgezogen, die erhaltene Lösung alkalisch gemacht und mit Perjodid gefällt. Das aus dem Niederschlag nach Stanĕks Vorschrift wiedergewonnene Chlorhydrat wurde über das Quecksilbersalz gereinigt und in das Chloraurat übergeführt. Dieses schmolz bei $264^{\circ}$ unter Zersetzung.

1) Diese Zeitschrift, Bd. 67, S. 86 .

2) 1. c. S. 76 . 


\section{0,5094 g Substanz gaben 0,2262 $\mathrm{g} \mathrm{Au}$.}

Für Cholinchloraurat $\mathrm{C}_{5} \mathrm{H}_{14} \mathrm{NOCl} \cdot \mathrm{AuCl}_{3}$.

Berechnet :

Gefunden:

$44,50 \% \mathrm{Au}$.

$44,40 \%$ Au.

Aus $1 \mathrm{~kg}$ lufttrockenem Insektenpulver konnten etwa $0,2 \mathrm{~g}$ Cholinchlorhydrat und 0,8 $\mathrm{g}$ Stachydrinchlorhydrat in reiner Form isoliert werden. ${ }^{1}$ )

II. Nachweis von Trigonellin in den oberirdischen Teilen von Mirabilis jalapa $\mathbf{L}$.

$600 \mathrm{~g}$ lufttrockenes Material wurde mit kochendem $70 \%$ igem Weingeist extrahiert, der Extrakt nach dem bekannten Verfahren (doppelte Fällung mit Phosphorwolframsäure nach vorausgegangener Reinigung durch Bleiessig) verarbeitet.

Die nach Zerlegung der Quecksilberdoppelsalze erhaltenen Chloride wurden gut getrocknet, dann zur Entfernung des Cholinchlorids mit kaltem, wasserfreiem Alkohol behandelt und auf eine Tonplatte gestrichen. Der Rückstand gab nach dem Umkrystallisieren aus Wasser glänzende Tafeln, deren Ausbeute $0,3 \mathrm{~g}$ betrug. Das salzsaure Salz wurde in das Chloraurat übergeführt, welches bei $196-197^{\circ}$ schmolz.

$$
0,3379 \mathrm{~g} \text { Substanz gaben } 0,1402 \mathrm{~g} \text { Gold. }
$$

Für Trigonellinchloraurat $\mathrm{C}_{7} \mathrm{H}_{7} \mathrm{NO}_{2} \cdot \mathrm{HCl} \cdot \mathrm{AuCl}_{3}$.

$$
\begin{array}{cc}
\text { Berechnet: } & \text { Gefunden: } \\
41,33 \% \mathrm{Au} & 41,49 \% \mathrm{Au} \text {. }
\end{array}
$$

Beim Umkrystallisieren aus Wasser verwandelte sich dieses Chloraurat in das basische Salz, welches bei $185-187^{\circ}$ schmolz.

Pikrat. Das, unseres Wissens, noch nicht dargestellte Pikrat wurde erhalten durch Versetzen einer wässerigen Lösung des salzsauren Salzes mit Natriumpikrat. Es bildet glänzende Prismen, welche in Wasser leicht, in absolutem Alkohol schwer, in Methylalkohol leicht löslich und in Äther fast unlöslich sind. Die Bestimmung des Pikrinsäuregehalts gab folgendes Resultat:

1) Die Frage, ob noch andere «Betaine vorhanden sind, muß vorläufig offen gelassen werden. Die Untersuchung der Chrysanthemumarten auf *Betaine» gedenkt der eine von uns (Dr. K. Yoshimura) in seiner Heimat fortzuführen. 


\section{$0,1676 \mathrm{~g}$ Substanz gaben $0,1042 \mathrm{~g}$ Pikrinsäure.}

Für Trigonellinpikrat $\mathrm{C}_{7} \mathrm{H}_{7} \mathrm{NO}_{2} \cdot \mathrm{C}_{6} \mathrm{H}_{3} \mathrm{~N}_{3} \mathrm{O}_{7}$.

\section{Berechnet:}

$62,57 \%$ Pikrinsäure.
Gefunden:

$62,77 \%$ Pikrinsäure.

Schmelzpunkt des Pikrats 198-200.

III. Nachweis von Cholin in Ajuga reptans L. (Oberirdische Teile.)

$375 \mathrm{~g}$ lufttrockenen Materials wurden mit 70\% igem Weingeist extrahiert und nach bekanntem Verfahren verarbeitet. Es wurden nur etwa $0,1 \mathrm{~g}$ Cholinchloraurat erhalten. Schmelzpunkt des Chloraurats 258-260 (unter Zersetzung).

\section{Nachweis von Cholin in käuflichen sFlores Rosmarinis.}

$4 \mathrm{~kg}$ lufttrockenen, zerkleinerten Materials wurden mit kochendem 70\% igem Alkohol extrahiert und die Extrakte nach dem wiederholt beschriebenen Verfahren aufgearbeitet.

Das nach Zerlegung der Quecksilberdoppelsalze erhaltene salzsaure Salz löste sich vollkommen in absolutem Alkohol. Nach dem Eindunsten auf dem Wasserbade und Stehenlassen im Exsikkator schieden sich zerfließliche Nadeln aus. Die Ausbeute an salzsaurem Salz betrug pro $1 \mathrm{~kg} 0,33 \mathrm{~g}$. Das salzsaure Salz wurde in das Chloraurat übergeführt, das bei $265^{\circ}$ unter Zersetzung schmolz.

0,2523 g Substanz gaben $0,1113 \mathrm{~g}$ Gold.

Für Cholinchloraurat $\mathrm{C}_{\mathbf{5}} \mathrm{H}_{14} \mathrm{NOCl} \cdot \mathrm{AuCl}_{3}$.

Berechnet:

$44,50 \% \mathrm{Au}$
Gefunden: $44,11 \% \mathrm{Au}$.

Pikrat. Das aus einem Teil des salzsauren Salzes dargestellte Pikrat, welches in Wasser leicht löslich war, schmolz bei $240-241^{\circ}$ unter Zersetzung.

\section{Nachweis von Cholin im Kraute von Glechoma hederacea L. (Nepeta} Glechoma Buth., Gundermann).

$4 \mathrm{~kg}$ der als Herba Hederae terrestris bezeichneten Droge wurden in gleicher Weise wie die eben beschriebenen Materialien verarbeitet. Nach Zerlegung der Quecksilberdoppelsalze wurden

Hoppe-Seyler's Zeitschrift f. physiol. Chemie. LXXVII. 
$0,8 \mathrm{~g}$ in absolutem Alkohol vollkommen lösliche Chloride pro $1 \mathrm{~kg}$ Ausgangsmaterial erhalten. Das Chloraurat schmolz bei $264^{\circ}$ unter Zersetzung.

$0,2736 \mathrm{~g}$ Substanz gaben $0,1209 \mathrm{~g}$ Gold.

Für Cholinchloraurat $\mathrm{C}_{5} \mathrm{H}_{14} \mathrm{NOCl} \cdot \mathrm{AuCl}_{3}$.

Berechnet:

Gefunden :

$44,50 \% \mathrm{Au}$

$44,19 \% \mathrm{Au}$.

VI. Nachweis von Cholin und 1-Stachydrin im Kraute von Galeopsis grandiflora Lam.

$4 \mathrm{~kg}$ der lufttrockenen Droge (Herba Galeopsidis grandifl. seu ochroleuc.) wurden mit Wasser extrahiert und nach bekanntem Verfahren verarbeitet.

Das nach Zerlegung der Quecksilberdoppelsalze erhaltene Chloridgemenge wurde mit wenig kaltem, absolutem Alkohol behandelt und auf eine Tonplatte verstrichen.

Der auf der Tonplatte verbliebene Rückstand bildete nach dem Umkrystallisieren aus Wasser feine Prismen, deren Ausbeute $1,65 \mathrm{~g}$ pro $1 \mathrm{~kg}$ Ausgangsmaterial betrug. Schmelzpunkt ca. $235^{\circ}$. Das Chlorid war in absolutem Alkohol ziemlich schwer löslich. Durch Behandeln von 1,5 g mit etwa $15 \mathrm{ccm}$ absolutem Alkohol ging nur 0,23 $\mathrm{g}$ in Lösung. Das Chlorid gab deutliche Pyrrolreaktion.

Optisches Drehungsvermögen des Chlorhydrats. a) 0,8000 g Substanz in $11 \mathrm{ccm}$ Wasser gelöst drehten im $2 \mathrm{dm}-\mathrm{Rohr}$ im Soleil-Ventzkeschen Apparat $-11,2^{\circ} \mathrm{S} . \mathrm{V}$.

$$
[\alpha]_{D}=-26,5^{\circ} \text {. }
$$

b) $0,3120 \mathrm{~g}$ Substanz wurden in $10 \mathrm{ccm}$ Wasser gelöst, sie drehten im $1 \mathrm{dm}-\mathrm{Rohr}-2,4^{0} \mathrm{~S} . \mathrm{V}$.

$$
[\alpha]_{\mathrm{D}}=-26,5^{\circ} \text {. }
$$

Die freie Base wurde durch Digerieren eines Teils des Chlorids mit feuchtem Silberoxyd dargestellt. Sie war hygroskopisch, in Wasser und Alkohol leicht löslich, hatte süßlichen Geschmack und reagierte neutral.

Chloraurat: Ein Teil des Chlorids wurde in das Chloraurat übergeführt. Schmelzpunkt $\left(225^{\circ}\right)$ und Aussehen unter dem Mikroskop stimmten mit Stachydrinaurat überein. Die Bestimmung des Goldgehalts gab folgende Resultate: 
Beiträge über das Vorkommen von Betainen im Pflanzenreich. 299

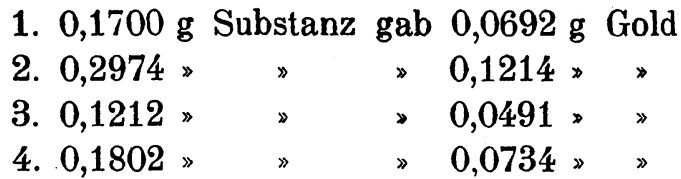

Für Stachydrinchloraurat $\mathrm{C}_{7} \mathrm{H}_{13} \mathrm{NO}_{2} \cdot \mathrm{HCl} \cdot \mathrm{AuCl}_{3}$.

Berechnet :

Gefunden :

$40,82 \% \mathrm{Au}$

1. $40,70 \% \mathrm{Au}$

2. $40,82 \% \mathrm{Au}$

3. $40,51 \% \mathrm{Au}$

4. $40,73 \% \mathrm{Au}$.

Auch der mit absolutem Alkohol in Lösung gegangene Anteil des Chlorids erwies sich als Stachydrinchlorid.

$0,1064 \mathrm{~g}$ Substanz gaben $0,0432 \mathrm{~g}$ Gold $=40,60 \% \mathrm{Au}$.

Pikrat, dargestellt aus dem Chlorid: Schmelzpunkt $195^{\circ}$.

Nachweis des Cholins. Zum Nachweis des Cholins dienten die im absoluten Alkohol leicht löslichen Anteile der aus der Sublimatfällung erhaltenen Chloride, bezw. die in die Tonplatte eingegangenen Anteile derselben. Die so erhaltene wässerige Lösung wurde nach Stan ̌̌ks Vorschrift mit Kaliumtrijodid in alkalischer Lösung behandelt und aus der entstandenen Fällung das Cholinchlorid erhalten. Ausbeute $0,50 \mathrm{~g}$ Chlorid. Das aus dem Chlorid dargestellte Chloraurat schmolz unter Zersetzung bei $262-263^{\circ}$.

$$
\text { 0,1702 g Substanz gab 0,0752 g Gold. }
$$

Für Cholinchloraurat $\mathrm{C}_{5} \mathrm{H}_{14} \mathrm{NOCl} \cdot \mathrm{AuCl}_{3}$.

Berechnet:

$44,50 \% \mathrm{Au}$
Gefunden: $44,18 \%$ Au.

Während das Phosphorwolframat des Stachydrins und anderer Betaine sich in sodaalkalischer Lösung glatt auflöst, ist das Phosphorwolframat des Cholins in sodaalkalischer Lösung nur unvollkommen löslich. Durch dieses Verhalten kann man die Gegenwart von Cholin neben Betainen ebenfalls erkennen.

VII. Nachweis von Stachydrin in Citronenschalen.

$1,5 \mathrm{~kg}$ des käuflichen lufttrockenen Materials (Cortex Citri Fructus) wurden zerkleinert und sodann mit 70\% igem Alkohol 
extrahiert. Die Extrakte wurden in gewohnter Weise verarbeitet. Das bei Zerlegung der Quecksilberdoppelsalze gewonnene Chloridgemenge wurde mit kaltem wasserfreiem Alkohol behandelt, wobei sich ein Teil (Cholinchlorid) löste. Der Rest wurde auf eine Tonplatte gestrichen, der zurückgebliebene Teil aus Wasser umkrystallisiert. Es wurden so etwa $0,3 \mathrm{~g} \mathrm{salz-}$ saures Salz erhalten. Das Salz gab deutlich die Pyrrolreaktion. Das Chloraurat stimmte in der Krystallform und im Schmelzpunkt $\left(225-226^{\circ}\right)$ mit Stachydrinchloraurat überein.

$0,3200 \mathrm{~g}$ Substanz gaben $0,1302 \mathrm{~g}$ Gold.

Für Stachydrinchloraurat $\mathrm{C}_{7} \mathrm{H}_{13} \mathrm{NO}_{2} \cdot \mathrm{HCl} \cdot \mathrm{AuCl}_{3}$.

Berechnet:

$40,82 \% \mathrm{Au}$
Gefunden: $40,69 \% \mathrm{Au}$.

Pikrat: Das aus dem Chloraurat regenerierte Chlorid wurde in das Pikrat übergeführt, welches gelbe Nadeln bildete und bei $195^{\circ}$ schmolz. Der Pikrinsäuregehalt war folgender:

0,1948 g Substanz gaben 0,1196 g Pikrinsäure.

Für Stachydrinpikrat $\mathrm{C}_{7} \mathrm{H}_{13} \mathrm{NO}_{2} \cdot \mathrm{C}_{6} \mathrm{H}_{3} \mathrm{~N}_{3} \mathrm{O}_{7}$

Berechnet:

Gefunden:

Pikrinsäure $61,56 \%$

$61,40 \%$.

Für die Bestimmung der optischen Aktivität reichte das Material nicht aus. In einer früheren Untersuchung hatten E. Schulze und G. Trier ${ }^{1}$ ) aus den Blättern der bitteren Orange inaktives Stachydrin erhalten. Herr Dr. N. T. Déléa no fand nun im hiesigen Laboratorium, bei einer in anderer Weise ausgeführten Isolierung des Stachydrins aus dem gleichen Material, die linksdrehende Form dieses Betains, und zwar stimmte die beobachtete spezifische Drehung mit der des Stachydrins aus Galeopsis (s. o.) überein. Die von Herrn Dr. Déléano ausgeführte Untersuchung hatte den Zweck, zu prüfen, ob sich die einfachere, auch im hiesigen Laboratorium von Prof. E. Schulze ${ }^{2}$ ) früher angewandte Methode der direkten Ausfällung von Cholin und eventuell vorhandener Betaine mit alkoholischer Sublimatlösung nach vorausgegangener Reinigung der Extrakte mit Blei-

1) Diese Zeitschrift, Bd. 67, S. 59 .

2) Siehe z. B. Diese Zeitschrift, Bd. 60, S. 157. 
Beiträge über das Vorkommen von Betainen im Pflanzenreich. 301

essig, speziell bei Stachydrin führenden Pflanzenteilen verwenden lasse. Von dieser Methode läßt sich im allgemeinen sagen, daß sie sich auch zur Gewinnung von Stachydrin aus Pflanzenextrakten verwenden läßt, daß man aber selbst bei mehrwöchentlichem Stehenlassen der Quecksilberfällungen kaum die Ausbeute erreichen wird, die man bei Verwendung von Phosphorwolframsäure erzielen kann.

Bei der Gewinnung von Stachydrin aus Orangenblättern erhielt Jahns ${ }^{1}$ ) unter Benützung der Kaliumwismutjodid-Methode eine Ausbeute von 0,1\% Stachydrin. Bei Benützung der Methode der doppelten Fällung mit Phosphorwolframsäure stieg die Ausbeute auf 0,19\%. Bei Benützung der Methode der direkten Sublimatfällung wurde die Ausbeute Jahns etwa erreicht. Es wurden aus $1 \mathrm{~kg}$ trockener Orangenblätter 1,25 g reines Stachydrinchlorhydrat gewonnen. Analyse des Goldsalzes:

$0,3710 \mathrm{~g}$ Substanz gaben 0,1514 g Au.

$\begin{array}{ll}\text { Berechnet: Gefunden: } & \\ 40,82 \% \mathrm{Au} & 40,80 \% \mathrm{Au} .\end{array}$

$0,5911 \mathrm{~g}$ Stachydrinchlorhydrat in $11 \mathrm{ccm}$ Wasser gelöst drehte bei $18^{\circ} \mathrm{C}$. $-8,2^{\circ} \mathrm{S} .-\mathrm{V}$. im $2 \mathrm{dm}$-Rohr

$$
[\alpha]_{\mathrm{D}}^{18^{\circ}}=-26,2^{\circ}
$$

Es war von Interesse, das Verhalten des 1-Stachydrins gegen Alkalien in der Hitze zu prüfen. Das Präparat wurde daher mit Barytwasser eine halbe Stunde gekocht und sodann das Stachydrinchlorid regeneriert. Das Salz war durch die Behandlung zum Teil racemisiert worden.

$0,5008 \mathrm{~g}$ Stachydrinchlorid in $10 \mathrm{ccm}$ Wasser gelöst drehte bei $18^{\circ} \mathrm{C}$. im $2 \mathrm{dm}$-Rohr $-2,7^{\circ} \mathrm{S} . \mathrm{V}$.

$$
[\alpha]_{D}^{18^{0}}=-9,3^{0} \text {. }
$$

Eine chemische Zersetzung war nicht erfolgt. Das Goldsalz, aus dem wiedergewonnenen Chlorhydrat dargestellt, erwies sich als einheitlich und gab den theoretischen Goldwert:

0,2764 g Substanz gab 0,1129 g Au $=40,84 \% \mathrm{Au}$.

Bei der Gewinnung des Stachydrins durch Fällung der gereinigten Extrakte mit alkoholischer Sublimatlösung wird die

1) Jahns, Ber. d. Deutsch. chem. Ges., Bd. 29, S. 2065. 
302 K. Yoshimura und G. Trier, Über Betainen im Pflanzenreich.

Einwirkung von Alkalien gänzlich vermieden. Man wäre vielleicht versucht, das Auftreten von inaktivem Stachydrin auf eine Racemisierung während der Verarbeitung zurückzuführen. Dagegen spricht jedoch einmal die Tatsache, daß bei Benützung der Methode der Phosphorwolframfällung die Einwirkung von Alkalien nur bei Zimmertemperatur stattfindet und daß bei Benützung des gleichen Verfahrens in mehreren Fällen zwar inaktives Stachydrin, wenigstens in einem Falle (Galeopsis), aber auch aktives Stachydrin erhalten wurde. Wir müssen also bis auf weiteres annehmen, daß sowohl linksdrehendes als auch inaktives Stachydrin in den Pflanzen selbst vorgebildet vorkommen kann.

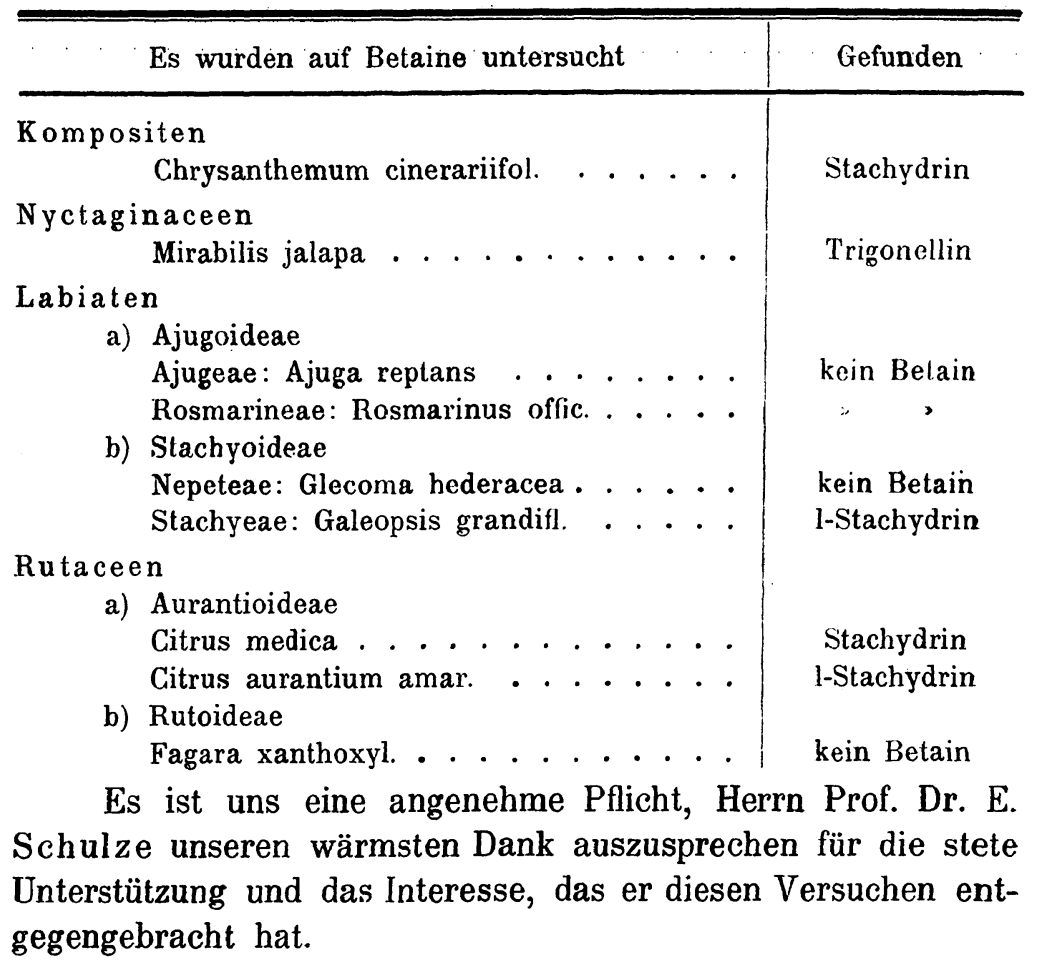

